EPJ Web of Conferences 47, 05009 (2013)

DOI: $10.1051 /$ epjconf/20134705009

(C) Owned by the authors, published by EDP Sciences, 2013

\title{
New spectrographs for precise RV at ESO
}

\author{
Luca Pasquini ${ }^{\mathrm{a}}$
}

ESO, Karl Schwarzschild str. 2, Germany

\begin{abstract}
Upgrades of existing spectrographs and plans for new instruments for precise radial velocity measurements at ESO are presented.
\end{abstract}

\section{INTRODUCTION}

Radial Velocity (RV) techniques have been proven to be very successful for the discovery and characterization of new extra solar planets. ESO has a long tradition of planet RV hunting, with several high resolution spectrographs (FEROS, [1], UVES, [3], HARPS, [2], CRIRES, [4] ) largely devoted to planet search and characterization.

Given the very high demand for these observations and the requests for improving RV precision in order to detect planets of lower mass, ESO is continuously improving the set of facilities capable to address this research. Such an effort evolves in two main directions: the continuous upgrade and improvement of the existing instruments, and the development of new facilities, for the $8 \mathrm{~m}$ VLT and for the new giant E-ELT.

In this contribution I provide a summary of these efforts.

\section{INSTRUMENT UPGRADES}

\subsection{HARPS}

The field of exoplanet search is evolving extremely fast, and almost every day a new planet is found. To date more than 700 extra-solar planets are detected, most of which $(>75 \%)$ by the radial velocity method. Thanks to the stability of HARPS, most precise measurements were obtained with this instrument, which allowed the detection of many of the super-Earth planets $\left(\mathrm{m}<10 \mathrm{~m}_{\text {Earth }}\right)$ and the lowest mass extra-solar planets, only 1.9 times the mass of the Earth (G1581e). As a reference, it should be considered that the Earth in its orbit causes a motion of the Sun around the common center of mass with a velocity of only $9 \mathrm{~cm} / \mathrm{s}$. The highest precision reached by HARPS, using Th-Ar lamps, is of $60 \mathrm{~cm} / \mathrm{s}$ on individual measurements. This precision ca be broken up in several components, out of which long term calibration stability and fibre scrambling are the dominant ones.

As the precision of the current instrumentation is increasing, the planetary mass function shows an emerging peak at low masses, which could be consistent with predictions from the core accretion theoretical model. From this model it is seen that the bulk of the planet population has masses below $20 \mathrm{~m}_{\text {Earth }}$ and it is yet below the detection threshold of most radial velocity surveys. The long term

\footnotetext{
${ }^{\mathrm{a}}$ e-mail: lpasquin@eso.org
}

This is an Open Access article distributed under the terms of the Creative Commons Attribution License 2.0, which permits unrestricted use, distribution, and reproduction in any medium, provided the original work is properly cited. 


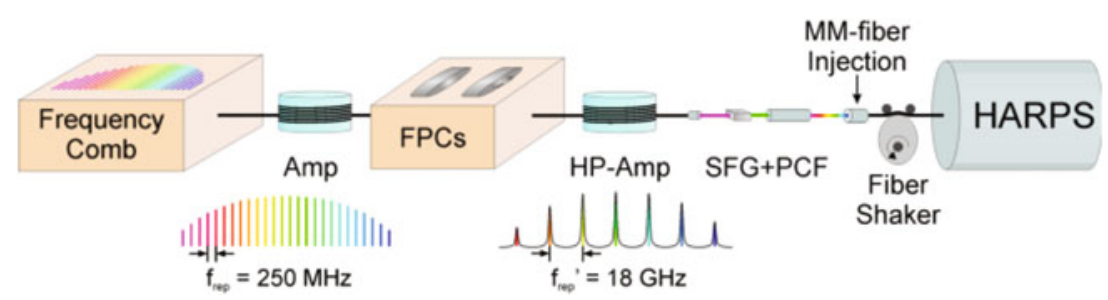

Figure 1. Scheme of the LFC system developed for HARPS. For the detailed description, see text. From [6].

precision of the wavelength calibration with the Th-Ar lamps is of $30 \mathrm{~cm} / \mathrm{s}$, and this is severely limiting the detection capabilities.

To gain access to the majority of these low-mass planets is necessary a new wavelength calibration method, with a precision better than $10 \mathrm{~cm} / \mathrm{s}$. This precision can only be reached if a Laser Frequency Comb (LFC) [5] is used as a wavelength calibration source.

LFCs bring the precision of the atomic clocks from the microwave domain, where the hyperfine transitions of $\mathrm{Cs}$ or Rb occur, to the visible or the NIR domain. In this way a spectrum can be generated, where each line is an integer multiple of the repetition frequency of the LFC, the latter being locked to an atomic clock:

$$
\Omega=\Omega_{0}+n \times \Omega_{R} .
$$

In the formula above, $\Omega_{0}$ is the offset frequency of the system, effectively a zero point, it is configurable and it is locked to the atomic clock; $\mathrm{n}$ is an integer and $\Omega_{R}$ is the repetition frequency of the LFC, also configurable and locked to the atomic clock. The accuracy of a modern atomic clock ranges from 1 part in $10^{11}$ typical for Rb clocks, to 1 part in $10^{16}$ for Cs clocks.

The scheme of the prototype already tested on HARPS is shown in Fig. 1 [6]. The High Repetition Rate (HRR) source output is centered at the fundamental frequency of the Yb-doped laser of $1030 \mathrm{~nm}$, and supplies lines with a spacing of $250 \mathrm{MHz}$. Two Fabry Perot cavities (FPCs) are used in series for mode suppression, achieving a final repetition rate of $18 \mathrm{GHz}$ and a suppression of more than $30 \mathrm{~dB}$. Amplifiers are inserted before and after the FPCs to increase the pulse energy. After frequency doubling (SFG), the LFC light is centered at $515 \mathrm{~nm}$ and covers a range of about $4 \mathrm{~nm}$. A Photonic Crystal Fiber (PCF) is used to broaden the spectral coverage of the comb via non-linear processes such as four-wave mixing, involving the redistribution of the energy to the different modes.

In collaboration with the MPQ group, ESO has started 5 years ago an R\&D program to produce a LFC for astronomical use, that has been tested on HARPS in several occasions [6, 7]. The technology is considered mature enough that a contract with an MPQ spinoff company, MENLO system, has been issued for the production of an LFC system for HARPS. The new system is expected to be delivered and commissioned at the end of 2013.

\subsection{CRIRES}

CRIRES is an echelle spectrograph for the $8 \mathrm{~m}$ VLT telescope, working in the IR regime. CRIRES design and use is not focussed for planet search, but it has been used quite often for this purpose by inserting a gas cell in the light path [9]. The interest of planet search in the IR domain has grown considerably in the last years, mostly because of the search around low mass, M stars. M stars have several advantages with respect to solar type objects. They have low mass, that implies that the RV signal is much larger for the same planet mass and period. M stars are cooler, therefore the habitable zone around them is much closer to the star. Both these arguments make that the discovery of rocky planets around M stars is much easier than around more massive objects. 


\section{Hot Planets and Cool Stars}

M stars are also the most common in the solar neighborhood, which implies that, once discovered, these planets will be the easiest to be studied in detail, given as well the lower contrast between the planet and star luminosity.

Since the M stars spectral energy distribution penalizes the visual and blue wavelengths, traditionally used for precise radial velocity search, the IR is the natural and most convenient way to observe them.

One of the main limitations of CRIRES is that the instrument is pre-dispersed, and only a tiny fraction of one echelle order is recorded at the time. Large spectral range is a huge advantage for radial velocity searches, because for a given $\mathrm{S} / \mathrm{N}$ ratio, the RV precision roughly scales with the inverse of the square root of the number of spectral lines. A larger spectral range will therefore bring an enormous advantage for these measurements. A large spectral range will also allow the simultaneous recording of many lines with a different sensitivity to atmospheric inhomogeneities, such as spots and active regions, allowing therefore to better disentangle between true planet signal and shifts induced e.g. by rotational modulation.

A phase A study for the modifications of CRIRES is carried out by a consortium that includes several institutes (PI: Dr. A. Hatzes, Tautemburg Observatory) and proposes a system of crossdispersers, a new detector array ad a polarimetric unit. A new system of gas cells will be able to allow precise radial velocity measurements up to the $\mathrm{K}$ band. If approved, in about 3 years CRIRES will be revamped and users will enjoy a new life for a very competitive instrument.

\section{FUTURE INSTRUMENTS}

\subsection{ESPRESSO}

ESPRESSO stands for Echelle SPectrograph for Rocky Exoplanets and Stable Spectroscopic Observations for the VLT. It combines the best properties of two leading instruments HARPS and UVES. On the one side it will enhance the stability of HARPS with an unprecedented resolution to obtain the best ever achieved Radial Velocity precision, on the other side it will be capable to collect the light from 4 VLT telescopes simultaneously, using for the first time a 16m diameter telescope, to greatly expand the VLT performances at faint objects [13, 14].

In order to understand the development of ESPRESSO, it is essential to briefly analyze the HARPS experience, and in particular the impact of HARPS in the discovery of low mass exo-planets. This is a new territory that is evolving very rapidly in the last years. In Section 1 the statistics of low mass planets is briefly discussed, but is not said that out of the few tens of exoplanets known today with a mass below 25 Earth Masses, the majority has been discovered with HARPS. The rate of these discoveries increases steadily and in the running high-precision program of HARPS it is estimated that more than $30 \%$ of the stars harbor extra-solar planets with masses below the mass of Neptune. What is perhaps even more interesting, is that two separate considerations suggest that there must be a huge amount of still undiscovered planets, even in already observed stellar samples. On the one hand, our limited precision induced an observational bias against small planet masses. On the other hand the prediction made by planet-formation models foresee a rapid growth of the number of planets at lower masses. Both these effects have been confirmed recently by a statistical analysis of the HARPS survey and by the large number of planet candidates found by the Kepler satellite. ESPRESSO is designed to explore this new domain and to enter into unknown territory by combining high efficiency with high instrumental precision. ESPRESSO will aim at detecting rocky planets of few Earth masses in the habitable zone of solar type stars. The use of the VLT large aperture will ensure the large number of photons needed to obtain the highest precision. Given its efficiency, spectral resolution and spectral domain, ESPRESSO will be most efficient up to M4 dwarfs, so it will be possible to use it also for the detection of planets around low mass stars. Finally. it will be ideal to carry out spectroscopic follow-up of Earth-sized planets discovered by the transit technique. By pushing the magnitude limits with respect to HARPS, more open clusters and star forming regions will become available for precise RV measurements. 
EPJ Web of Conferences

\begin{tabular}{|c|c|c|c|}
\hline Parameter & singleUHR & singleHR & multiMR \\
\hline Wavelengths & \multicolumn{3}{|c|}{$\begin{array}{l}\text { Blue arm: } 380-520 \mathrm{~nm} \\
\text { Red arm: } 520-780 \mathrm{~nm}\end{array}$} \\
\hline Spectral coverage & \multicolumn{3}{|c|}{ Full } \\
\hline Spectra format & \multicolumn{3}{|c|}{ Up to 4 spectra per order ( 1 object +1 reference fibers, 2 spectra / fiber) } \\
\hline Resolving power & $200 ’ 000$ & $130 ’ 000$ & $55^{\prime} 000$ \\
\hline Aperture on sky & 0.5 arcsec & $1.0 \operatorname{arcsec}$ & $4 \times 1.0$ arcsec \\
\hline Spectral sampling & $>2$ pixels & $>3.5$ pixels & $>4$ pixels (binned $\times 2$ ) \\
\hline Spatial sampling & $>4$ pixels & $>8$ pixels & $>5$ pixels (binned $x 4$ ) \\
\hline Sky/ Simultaneous reference & \multicolumn{3}{|c|}{ Yes (mutually exclusive) } \\
\hline Instrumental RV precision & $<10 \mathrm{~cm} / \mathrm{s}$ & $<10 \mathrm{~cm} / \mathrm{s}$ & $<1 \mathrm{~m} / \mathrm{s}$ \\
\hline
\end{tabular}

Figure 2. Summary of the instrument modes and capabilities for ESPRESSO. From [11].

To bring the light form the telescopes to the incoherent focus coude' room a coude' train is needed; the light is then collected by several fibre systems, that allow range of resolving powers and combinations, going from $\mathrm{R}=220000$ with one telescope, to the $\mathrm{R}=60000$ with the 4 telescopes simultaneously, as summarized in Fig. 2 (from [11]). All these modes are possible without having moving parts in the spectrograph, that is temperature and pressure controlled. A full description of ESPRESSO and its main subcomponents can be found in [11], and references therein. ESPRESSO it will be available in 2016 at the VLT telescope.

An analysis of the HARPS experience and error budget has been used to guide the design and to improve the performances. The required (and expected) RV precision of ESPRESSO with 1 UT telescope is of $10 \mathrm{cms}^{-1}$.

\subsection{E-ELT}

The European ELT (E-ELT) is the next major ESO project. Several years ago ESO launched a series of Phase A studies for E-ELT instruments, and two high resolution spectrographs that aimed at planet searches were proposed: CODEX [10] in the optical domain and SIMPLE [12] in the IR. Driven by the direct measurement of the expansion of the universe [8] and by the discovery of earth-like planets, CODEX aimed at the highest RV precision, $1-2 \mathrm{cms}^{-1}$. SIMPLE was mostly centered in the study of planets around low mass stars and their characterization.

Recently a conference on "High Resolution Spectroscopy" with the E-ELT has been organized at Cambridge (UK), by R. Maiolino and M. Haenhelt, aiming at discussing the scientific cases of high resolution spectroscopy with the E-ELT, to summarize the top level requirements emerging for such an instrument by a considerable fraction of community of users. Planet characterization is definitely at the top of the scientific interests. The high resolution spectrograph has been inserted in the E-ELT instrumentation roadmap as fourth or fifth instrument (https://www.eso.org/sci/. facilities/eelt/instrumentation/) and its scientific requirements are also discussed at the conference ESO - E-ELT Science \& Instrumentation in February 2013.

The instrumentation park at ESO for precise RV seems to have therefore a great present and a brilliant future.

\section{References}

[1] Kaufer, A. et al., The Messenger 95, 8 (1999)

[2] Mayor, M. et al., The Messenger 114, 20 (2003)

[3] Dekker, H. et al., Proceedings SPIE 4008, 534 (2000)

[4] Kaufl, H.-U. et al., Proceedings SPIE 5492, 1218 (2004) 


\section{Hot Planets and Cool Stars}

[5] Steinmetz, T. ., et al. Science 321, 1335 (2008)

[6] Wilken, T. ., et al. Nature 485, 611 (2012)

[7] Willken, T. ., et al. MNRAS 405, L16 (2010)

[8] Liske, J., et al. MNRAS 386, 1192 (2008)

[9] Bean, J.L., et al. ApJ 711, L19 (2010)

[10] Pasquini, L. et al., The Messenger 122, 10 (2005)

[11] Megevand, D. et al., Proceedings SPIE 8446, 1 (2012)

[12] Origlia, L. et al., The Messenger 140, 38 (2010)

[13] Pepe, F. et al., Proceedings SPIE 7735, 14 (2010)

[14] Pasquini, L. et al., Science with the VLT in the ELT Era Springer, Netherlands, 2009, p. 395 